BULLETIN Bulletin hispanique

HISPANIQUE Université Michel de Montaigne Bordeaux

$116-2$ | 2014

Référentialité/autoréférentialité dans le roman espagnol contemporain : bilan et perspectives

\title{
La voz de Ofelia
}

Une voix déployée

Évelyne Martin Hernandez

\section{(2) OpenEdition}

\section{Journals}

Édition électronique

URL : http://journals.openedition.org/bulletinhispanique/3392

DOI : 10.4000/bulletinhispanique.3392

ISBN : 979-10-300-0156-3

ISSN : $1775-3821$

Éditeur

Presses universitaires de Bordeaux

\section{Édition imprimée}

Date de publication : 1 décembre 2014

Pagination : $563-570$

ISBN : 978-2-86781-963-6

ISSN : 0007-4640

Référence électronique

Évelyne Martin Hernandez, "La voz de Ofelia », Bulletin hispanique [En ligne], 116-2 | 2014, mis en ligne le 01 décembre 2017, consulté le 20 avril 2019. URL : http://journals.openedition.org/

bulletinhispanique/3392 ; DOI : 10.4000/bulletinhispanique.3392 


\title{
La voz de Ofelia. Une voix déployée
}

\author{
Évelyne Martin Hernandez \\ Université de Clermont Ferrand
}

Dans La voz de Ofelia, Clara Janés relate sa rencontre avec le poète tchèque Vladimir Holan. Le verbe replegar(s)e, réitéré dans ce récit autofictionnel, peut en caractériser la structure complexe qui, par ses plis, replis, déplis, rend compte des surprenantes concordances entre les vies et les écrits des deux protagonistes. Ce faisant, le texte éclaire également le surgissement de la voix et, tout en en préservant le mystère, le travail de la création poétique.

Mots-clés : Clara Janés, Vladimir Holan, poésie, création, structure en plis.

En La voz de Ofelia, Clara Janés evoca su encuentro con el poeta checo, Vladimir Holan. El verbo replegar(se) que se repite a lo largo del relato autoficcional, puede dar una clave de su compleja estructura, ya que sus pliegues, repliegues, despliegues, dan cuenta de las sorprendentes concordancias entre vidas y escritos de los dos protagonistas. El texto, además, arroja luz sobre el brotar de la voz y, sin por ello desvelar su misterio, el trabajo de la creación poética.

Palabras claves: Clara Janés, Vladimir Holan, poesía, creación, estructura en pliegues.

In La voz de Ofelia, Clara Janés relates her encounter with Czech poet Vladimir Holan. The verb replegar(se), recurrent in this self-fiction, emblematizes its complex structure which, in its recourse to folding, doublefolding and unfolding, displays the surprising correspondences between the lives and writings of the two characters. At the same time, the text also reveals the emergence of the voice and the labour of poetic creation, while preserving its mystery.

Keywords: Clara Janés, Vladimir Holan, poetry, creation, fold structure. 

a création de ce livre ${ }^{1}$ a une longue histoire que Clara Janés résume dans
une Nota finale :

La escritura de este libro se remonta, en sus orígenes, a más de una década. En aquellos años, la tentativa de contar mi encuentro con el poeta checo Vladimir Holan me situó ante la figura de mi padre y su muerte, suceso fundamental de un libro de memorias de infancia $y$ adolescencia por entonces inacabado. El texto naciente, que desde un principio se llamó La voz de Ofelia, asaltó a aquel primero, aún sin título, y ambos iniciaron un intercambio. La voz de Ofelia empujó al otro a cobrar su forma definitiva que publiqué, en 1999, con el nombre de Jardín y laberinto (p. 107).

Outre ce curieux dialogue initial entre les deux ouvrages, il y eut une étape intermédiaire, le projet d'un monologue que l'actrice Janine Mestre désirait monter. L'auteure était en scène, elle écrivait ses mémoires et la première version de la pièce s'achevait sur le planctus du livre Vivir², le chant funèbre dédié à son père, mort dans un accident. Dans une deuxième version, le monologue incluait plusieurs poèmes ${ }^{3}$. C'est après l'abandon de ce projet théâtral et quelque douze années de "décantation" que les deux livres furent achevés.

La voz de Ofelia est donc le récit de la rencontre, une rencontre-choc, révélatrice, avec, dans un premier temps, un long poème traduit en espagnol : Una noche con Hamlet, puis, après quelques échanges épistolaires, avec son auteur, le poète tchèque Vladimir Holan ${ }^{4}$. Clara Janés évoquait en 2005 à Prague les événements qui inspirèrent ce deuxième livre de souvenirs :

En un momento dado, yo le dediqué un poema, lo mandé a su traductor, él se lo tradujo y se creó el primer nexo. El me mandó un libro. $Y$ a partir de ahi yo pude ir a verle. Era todo muy enigmático. Yo le visité en 75 , en 77 hasta el 79 . Y yo veía que algo sucedia, y que era muy fuerte también en su relación conmigo, no solamente en mi relación con su poesía. Y no podía explicármelo. Sólo al final, cuando me dejaron conocer el poema Una noche con Ofelia, entendi lo que sucedía. En el poema de Holan, Ofelia era como yo de Barcelona, hacia el viaje a Bohemia para conocer a Mácha y acontecían varias cosas que yo misma habia vivido. Y el caso es que Holan habia escrito el poema antes de conocerme, antes de saber que yo existía y antes de que yo intentara entrar en contacto con él, pero en el tiempo en que yo llevaba ya un año leyendo su libro. Este es un hecho tan enigmático, tan extraordinario que necesité doce años para saber cómo contarlos.

Pour le lecteur, la première énigme que présente le récit, c'est sa composition, souvent caractérisée comme circulaire, parfois comme chaotique ${ }^{6}$, obéissant au seul hasard du surgissement des souvenirs, lequel n'est pas censé respecter la chronologie...

1. La voz de Ofelia, Madrid, Siruela, Libros del tiempo, 2005.

2. Madrid, Hiperión, 1983.

3. Information recueillie dans une conversation téléphonique avec Clara Janés le 28/08/2012.

4. Vladimir Holan, 1907-1980, est un poète tchèque, d'abord avant-gardiste, influencé par Valéry et Rilke, puis engagé dans la résistance au nazisme, et qui, après avoir été longtemps interdit de publication par les gouvernements communistes, a vécu reclus dans sa maison de l'île de Kampa située entre les bras de la Vlatva (la Moldau), à Prague.

5. Intervention à la radio tchèque, citée par Hedvika Vydrová, "Clara Janés y Vladimir Holan: el secreto de la palabra poética", in Opera románica, n 8, Ed. Universitatis Bohemiae Meridionalis, 2005, document aimablement communiqué par Clara Janés.

6. Voir les travaux de Ana Pasero, Ana Casas Aguilar, Michèle Ramond, Nadia Mekouar, qui sont plus centrés sur le genre, l'autobiographie féminine, la relation au père, à la mère. 
Il s'agit d'une suite de pièces brèves, mais d'importance inégale, d'une phrase (parfois même inachevée) à trois pages, exception faite de la dernière qui est plus longue. Comme des feuillets accumulés, empilés au fil des jours, des mois, des années et qui rapportent aussi bien des souvenirs que des méditations sur la création, des extraits du premier volet de souvenirs, Jardín y Laberinto ${ }^{7}$, des poèmes du livre $K a m p a^{8}$; certaines pages incluent des citations de Holan, surtout, mais aussi, plus succinctement, de Rilke, de Shakespeare. On a affaire à un ensemble hétérogène dont l'agencement n'est peut-être pas pour autant aléatoire.

Un autre élément qui peut surprendre est la disparité des 2 parties qui composent le texte : la première qui comprend 57 séquences couvre 88 pages et la $2^{\mathrm{e}}$ partie une seule séquence de 5 pages.

Plusieurs thèmes traversent le livre et relient ces différentes séquences : la nature et en particulier les fleurs, les roses, la musique et le chant, un buste de femme dans la maison du poète, ainsi que la récurrence fréquente du verbe "replegar", ou "replegarse ", dont le sens varie. Il peut désigner l'état d'esprit ou la réaction de celle qui parle, mais aussi la situation de Holan dans Prague.

D'où la tentation d'examiner cette forme du repli et du pli et d'examiner si, en dehors du contexte psychologique ou comportemental, elle ne pouvait pas s'appliquer à la construction même du texte, à la manière dont elle le façonne, selon diverses opérations : plis, déplis, replis, etc. Il fallait voir si l'introspection, "la conciencia, este conocimiento vuelto sobre si mismo" ", ne s'inscrivait pas structurellement dans la production du discours par des retours en arrière, des rebroussements, des reprises, des rabattements d'épisodes les uns sur les autres, des inclusions, lesquelles enveloppent un élément important comme le pli confidentiel le ferait pour une information capitale, etc.

Un premier niveau de "pliage " pourrait correspondre à celui du pliage ou plissé en accordéon, car ces séquences disparates, tant par la longueur que par la nature, donnent lieu à un enchaînement quasi ininterrompu du récit. Elles sont en effet reliées l'une à l'autre par ce que, dans la lyrique médiévale galicienne, on appelle le leixapren. La fin d'une strophe, ici d'une séquence, est répétée au début de la suivante. Qu'il s'agisse d'une phrase, d'un substantif, d'un motif, le résultat est le même : le courant de pensée, suivant le fil des souvenirs et les réflexions que ceuxci engendrent, rebondit comme sur les différentes faces d'un rouleau de papier plié en accordéon, et les jointures-pliures sont constituées par ces réitérations ${ }^{10}$.

7. Madrid, Debate, «Literatura », 1990.

8. Madrid, Hiperión, 1986.

9. Clara Janés, La palabra y el secreto, Madrid, Huerga y Fierro, "La rama dorada ", 1999, p. 93 (Extrait d'un passage du "Viaje nocturno ", où elle analyse la poésie de Holan et en reprend certains thèmes).

10. Deux exemples : la séquence $n^{\circ} 5$ reproduit un dialogue entre Orphée et Eurydice, du texte de Holan, la séquence $n^{\circ} 6$ évoque l'air "Che faro senza Euridice", chanté par la mère de la narratrice. La notion de "arrojo", sur laquelle se clôt la page 26 : "es necesario tener ese valor, ese arrojo ", et par laquelle s'ouvre la page 27 : "Fue también su arrojo lo que fecundó mi admiración por él $[\ldots] »$. 
Le texte travaille ainsi à reproduire ce que C. Janés appelle, en citant Rosa Chacel, «la cohesión de la memoria ${ }^{11}$ ".

À lire ces enchaînements, on pourrait penser que c'est par une simple association d'idées, de mots ou images, que se produit l'affleurement des souvenirs et leur succession. Or, on peut déceler un autre mécanisme à l'œuvre dans l'articulation des séquences entre elles, celui qui consiste à rabattre un épisode sur un autre, voire à le dépasser, en revenant en amont du premier par chevauchement de celui-ci. C'est ce qui se produit dès le début du récit puisque la première séquence expose le lieu de la réception d'une lettre dont on ne saisit l'importance pour celle qui parle qu'à la lumière du contenu de la troisième séquence: "Sí, tras seis años de abandono de la poesía, seis años de hallarme en estado latente, como la semilla en su cápsula, bajo una tierra helada, yo iba a mi ser, iba a mi propio ser, por la palabra de aquel poeta checo ${ }^{12}$ ".

Or, la lettre, arrivée à Barcelone, est une réponse à un envoi effectué à Paris ${ }^{13}$, ce qui donne lieu à une interrogation, plusieurs fois formulée, sur cette énigme. Une interrogation qui fait ainsi retour sur l'événement. Et tout au long du récit, ce type de questionnement se produira, par un effet de réflexivité qui dédouble les épisodes en les complexifiant.

Par ailleurs, le lieu de naissance, Barcelone, convoqué par cette lettre, ne va pas cesser de se manifester, de revenir à l'esprit de la narratrice : "Vuelve Barcelona y vuelve Pedralbes... ${ }^{14}$ ", "Pero la ciudad vuelve... ${ }^{15}$ ", de sorte que les souvenirs d'enfance vont se déployer comme spontanément, avec parfois de curieux effets d'écho et de mise en abyme, car certaines lignes (comme celles qui viennent d'être citées) sont empruntées à Jardín y laberinto, voire à des versions préparatoires de ce livre. Le retour du passé s'accompagne d'un retour sur le passé, par une pratique constante de la enmendatio, qui permet de dévoiler peu à peu ce qu'une scène initiale "implique " dans tous les sens du verbe $\mathrm{y}$ compris celui de sa racine latine, (implicare : plier, entortiller, emmêler). Jouée et rejouée, cette scène met en lumière des coïncidences décisives, en particulier dans et par l'espace.

En effet, pour celle qui parle, revenir à Pedralbes, à la Barcelone de l'enfance, au jardin de la maison familiale, c'est refaire le chemin qui mène inéluctablement à la rencontre avec Holan, par la paradoxale similitude des lieux auxquels ils sont tous deux, d'une façon ou d'une autre, attachés. Pedralbes, la maison familiale et son jardin sont l'image d'un vert paradis, lumineux, clos, abrité du monde, lequel apparaît au loin, comme une promesse. On y perçoit aussi le chant des clarisses du couvent voisin, ce qui redouble le thème de la clôture. Kampa, où est reclus Holan, est le nom d'une véritable île de la Vltava dans Prague; de plus le poète s'y est volontairement enfermé, volets clos, depuis sa mise à l'écart de la

11. La palabra y el secreto, ed. citée, p. 24.

12. La voz de Ofelia, p. 18.

13. C. Janés avait décidé d'apprendre le tchèque pour lire Holan dans le texte et le traduire, ce qu'elle fit à Paris, à l'Institut des Langues Orientales.

14. La voz de Ofelia, p. 25

15. Ibidem, p. 28 
vie publique par le régime. Il y a donc une forte analogie entre ces deux espaces, isolés, où les enfermements s'emboîtent comme des tables gigognes, "muro por muro ", dit le texte janesien, en citant Holan. Cette " pré-disposition » des lieux à la complicité entre les deux personnages est rappelée à plusieurs reprises, elle sera plus tard explicitée : "Sólo la de Pedralbes pudo llegar a ser la de Praga ${ }^{16}$ ". Et elle justifie a posteriori l'émotion qu'éprouva la protagoniste en découvrant le poème Una noche con Hamlet, ainsi que sa fascination "cada vez que se abría el libro ", exposée dans les premières séquences du récit. Sa lecture produit deux effets, un effet curatif: «se me iba llevando, me sacaba de los infiernos" (p.19), et un effet de révélation par reconnaissance, par spécularité : "el libro expresaba el recorrido de mi propio cerebro", "los mismos caminos de mi laberinto ${ }^{17}$ "(p. 32), "Como los versos de Holan reflejaban [...]" (p. 38). Elle préfigure également le dialogue entre les deux poètes, d'abord par un échange de lettres et de poèmes, puis lors des visites que la narratrice fera à l'auteur tchèque, alors déjà très malade.

Le rappel, dans cette première partie du livre, de la mort accidentelle du père, signe l'arrachement au monde protégé de Pedralbes. Cependant, lorsque cette disparition est remémorée, elle trouve une étrange résonance dans le texte de Holan dont la protagoniste ne se sépare pas : "estaba en el autobus, con el espectro de Hamlet " (p. 18), Hamlet, lui aussi "inconsolable ", pour citer Shakespeare. Cette période correspond de plus, pour elle, à la fin d'une relation amoureuse. Il s'ensuit l'évocation d'une souffrance dont les symptômes se confondent avec le comportement du personnage d'Ophélie :

Go to a nunnery, dijo entonces la ciudad... y ya me replegaba una vez más, para abandonarme a esta corriente secreta, orillada de flores silvestres, cauce a mi desamparo, en pos de una certeza, acaso también yo bajo una cúpula trazada por las guedejas de un sauce... ${ }^{18}$.

On reconnaît dans ce passage l'errance désespérée de l'héroïne shakespearienne, cruellement rejetée par Hamlet, or celle qui parle est à ce moment du récit tentée par la réclusion dans la folie ${ }^{19}$ ou l'anéantissement.

"Pero en Barcelona estaba también el libro... " : le récit s'infléchit ${ }^{20}$ ici, car Barcelone présente deux visages : celui de la perte et celui de la brèche qu'ouvre la lecture du livre Una noche con Hamlet dans « l'enfer-mement » de la mélancolie.

L'évocation du souvenir de cette lecture permet l'expression de divers états d'âme, en suscitant une sorte de ballet de spectres. Ce ne sont pas seulement le père mort et le poète tchèque qui va mourir, mais les héros littéraires et mythiques avec lesquels l'instance d'énonciation s'identifie et identifie le poète

16. Ibidem, p. 50

17. Ibidem, p. 32

18. Ibidem, p. 40.

19. La parenté de la protagoniste avec l'hérö̈ne de Shakespeare est soulignée par la citation $\mathrm{du}$ texte et le nunnery anglais est en résonance avec le couvent de Pedralbes dont les chants parvenaient jusqu'au jardin de l'enfance de la narratrice.

20. Ici aussi, on peut se référer à la racine latine : inflectere : plier. 
admiré. Orphée s’incarne dans Vladimir Holan, «se me iba llevando, me sacaba de los infiernos" (p.19). Et, curieusement, c'est lui que le poète tchèque fait dialoguer avec Eurydice dans le passage de Una noche con Hamlet inclus au début du texte de Janés ( $2^{\mathrm{e}}$ séquence). Hamlet est mis en scène et c'est Orphée qui parle, d'où le chassé-croisé des personnages Hamlet-Ophélie, avec OrphéeEurydice, incarnés tour à tour par les deux protagonistes tout au long de $L a$ voz de Ofelia, mis en œuvre par un jeu de parallélismes, d'oppositions, de chiasmes. Il est difficile, dans une étude brève, de rendre compte de la mobilité, de la labilité de ces figures et de leur évolution au gré des plis et replis du texte qui font passer de la littérature à la vie et vice versa dans une sorte de " transfusion ${ }^{21}$ opérée dans les deux sens. Clara Janés évoque, dans La palabra y el secreto, ce pouvoir de "trasvase de la vida a la poesía " ${ }^{22}$, en commentant une fois encore Vladimir Holan. Je n'en donnerai que deux exemples.

Le poète (Holan), d'abord présenté comme un Orphée capable de sauver Eurydice est aussi assimilé à ses personnages : "Asi Hamlet, asi Ofelia. Y su reverso: la que es rescatada de la muerte. Y yo, al leer su poesía, tras seis años de esterilidad, fui también rescatada» (p. 27). La même formule elliptique donne à la protagoniste deux visages (ou rôles) masculins : "Asi pues, Orfeo y Hamlet", l'un portant sa (ses) certitude(s) et l'autre son (ses) doute(s) (p. 29). Il est tentant d'établir une homologie entre les différentes facettes des personnages, les unes constituant le revers des autres et la structure du pli qui présente deux aspects d'une même surface, l'un éclairé et l'autre dans l'ombre (le vivifiant, revivifiant vs le mortifère). Cette mouvante distribution des rôles de héros mythiques fait se déplier, se déployer et se multiplier les convergences ou concordances entre les deux protagonistes.

Mais c'est bien entendu la figure d'Ophélie que l'on retrouve le plus souvent dans ce récit. Et son identification avec la protagoniste, avant d'être " corroborée " par la révélation finale, repose sur de multiples échos du texte anglais. On a vu plus haut les allusions aux guirlandes de fleurs que tressait en chantant l'héroïne de Shakespeare, avant de les accrocher aux branches d'un saule, pour être enfin emportée par la rivière. Ces motifs, souvent discrètement évoqués -il faudrait y ajouter ces eaux souterraines auxquelles la protagoniste songe à s'abandonner- dessinent et redessinent la double figure féminine. Double ou triple, car la fin du récit, la deuxième partie, qui se rabat sur la première, résout (déplie) les énigmes que celle-ci posait ou suggérait, en fixant un dernier portrait d'Ophélie. Holan, dans un deuxième long poème, Una noche con Ofelia, avait imaginé une hérö̈ne et elle est venue s'incarner devant lui en la personne de la protagoniste. Ce texte, la narratrice ne le découvre qu'après la mort du poète tchèque, et c'est une sorte de coup de théâtre qui est inscrit dans la dernière séquence du récit. Elle, de son côté, après s'être

21. Cf. le rêve, état intermédiaire, où la protagoniste éprouve le désir de recevoir, par transfusion, non seulement encore le sang, mais la chair et les os de Holan (op. cit., p. 71).

22. La palabra y el secreto, p. 92. 
identifiée avec différents personnages, égarée qu'elle était par les tribulations de la vie, se reconnaît dans cette vision prémonitoire que le poète-voyant a eu du personnage d'Ophélie, une Ophélie catalane, son double ${ }^{23}$, sa réplique ${ }^{24}$ "rescatada", comme l'Eurydice de Una noche con Hamlet. La figure de deuil s'inverse alors en figure de renaissance : " todo en míse modificaba, se reorientaba " (p. 102). La littérature est donc ici comme un simulacre anticipé de la vie (ou bien la vie est-elle un décalque tardif de la littérature ?).

Cette Eurydice est par ailleurs deux fois sauvée par Orphée-Holan, la première étant le moment où la lecture de Una noche con Hamlet l'arrache à la mélancolie dans laquelle elle se trouve, comme le préfigurait un passage précédemment cité : "replegada en mi misma, como la semilla en su cápsula, bajo una tierra helada, yo iba a ser, iba a mi propio ser por las palabras de aquel poeta checo " (p. 18). La métaphore de la graine enclose suivie de l'éclosion de la fleur dont elle était porteuse, puis celle de la chrysalide sur le point de se déplier en papillon (p. 75), sont en parfaite résonance avec la construction en plis et déploiements du texte et avec son sens : la métamorphose de celle qui parle et l'épiphanie de la poésie ${ }^{25}$.

La deuxième forme d'accomplissement de cette Ophélie-Eurydice-Orphée est, paradoxalement, la capacité à trouver dans le repli, la profondeur et le secret, la source de l'expression la plus intense : le chant. C'est La voz de Ofelia, celle dont est relaté le jaillissement dans plusieurs passages.

Le texte suit le travail d'exploration de l'intime, de l'espace intérieur, qui, comme dans l'œuvre du poète tchèque, enferme «l'avant ", l'origine, l'espace abrité par la nuit, l'isolement, où la naissance et la mort se conjuguent, où l'invisible est perçu et qui à plusieurs reprises apparaît dans le livre comme "repliegue ":

"cuanto alberga los más recónditos repliegues del corazón [...] " (p. 40), todo significando mucho más, irradiando, y todo en ese repliegue oculto en el que hay que indagar porque hay que ir cada vez más hondo hasta que la mirada, dejando atrás Pedralbes, cruce la sombra prenatal y alcance el primer destello de vida y uno mismo se convierta en su propio Orfeo (p. 26).

La plus étonnante de ces métaphores qui tournent autour du pli, du repli ou de l'enveloppe est celle où est évoquée la part secrète et indestructible de la parole posthume du poète (p. 88 : "es él mismo, el corazón humano latiendo, despojado de sus envolturas - pecho, entraña, membrana - ", avec une référence implicite à Ibn Arabi, mais qui fait aussi penser aux replis de l'âme, de Leibniz, dont parle si bien Deleuze ${ }^{26}$.

23. L'Ophélie de Holan est de Barcelone, elle sort violemment du Palau, elle met sur la table des "pechinas, veneras y conchas", cadeau que la narratrice avait préparé pour sa dernière visite frustrée au poète.

24. Au sens de "reproduction ", de " double " (au XIX" siècle, selon Le Robert Historique de la Langue Française), sans compter le sens latin de replicare : replier...

25. "La nueva persona se estaba definiendo", p. 72.

26. Gilles Deleuze, Le pli. Leibniz et le baroque, Paris, ed. de Minuit, 1988. 
Le pli, pour conclure, semble bien être dans ce récit la forme qui met en évidence les coïncidences insolites. Par une mouvante superposition des espaces et des temps, qui en déplace l'ordre habituel, cette forme fait entendre les résonances, les consonances, les concordances qui rapprochent deux êtres, deux voix poétiques. C'est aussi le révélateur d'un mystère dont il préserve cependant, de façon métaphorique, le secret : celui de la création. Autrement dit, et avec plus de légèreté, par Francis Ponge : «Il ne s'agit que de selfspliquer les choses (ou tour à tour chaque chose) dans leur complétude énigmatique ${ }^{27}$ ». 